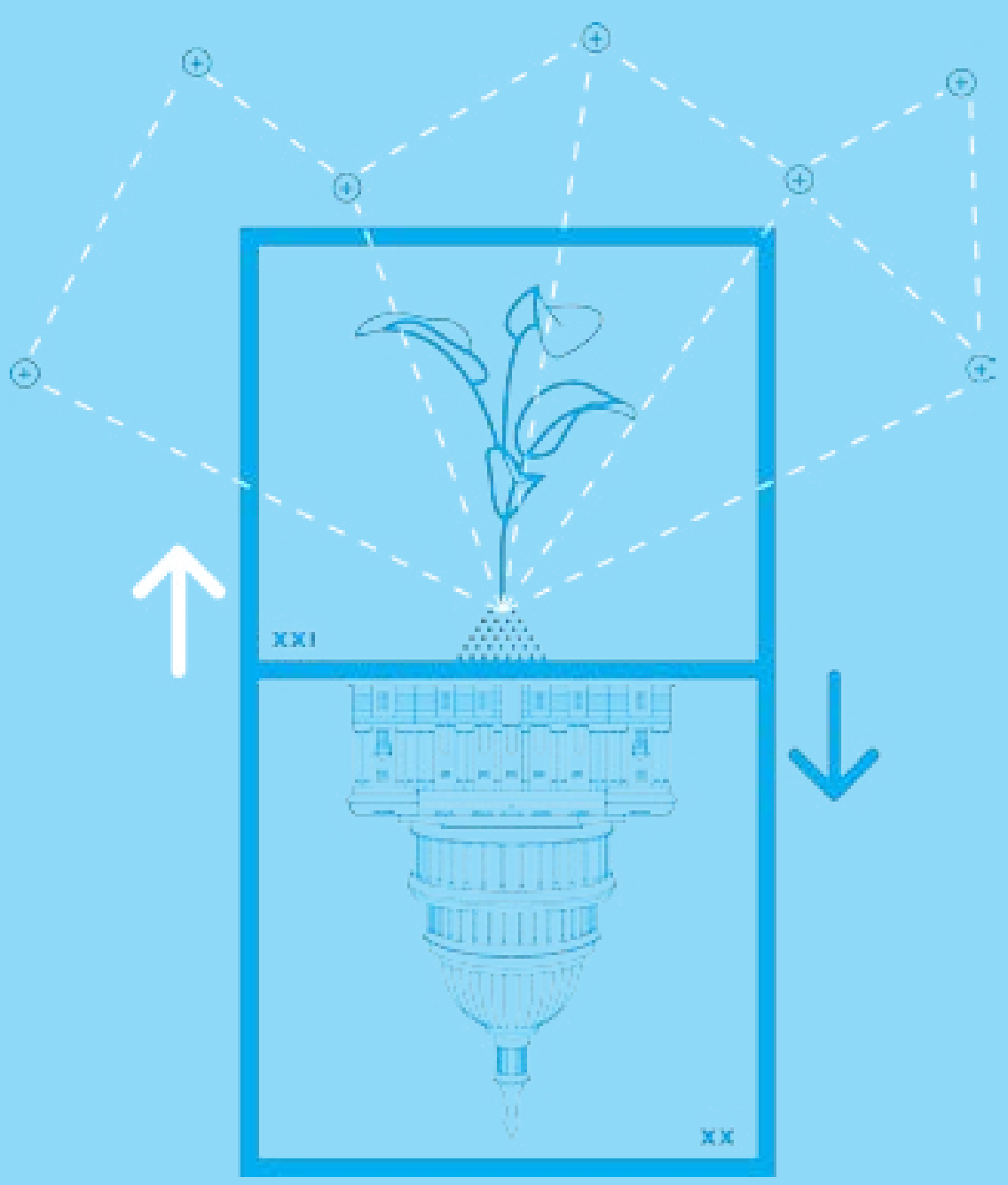

\title{
Nuevos sentidos y alternativas para la educación en contextos de transformación
}

New ways and alternatives to education in contexts of transformation

Novas formas e alternativas para a educação em contextos de transformação 


\section{Carlos Crespo Burgos ${ }^{1}$}

1 Master en Ciencias Sociales Aplicadas a la Educación, Facultad de Educación, Universidad Estadual de Campinas, Sao Paulo, Brasil. Realiza sus estudios de Doctorado en Educación en la Facultad de Educación de la Universidad Estadual de Minas Gerais, Belo Horizonte, Brasil.Actualmente es Director del Centro Nacional de Investigaciones Sociales y Educativas del Ecuador (CENAISE). Profesor del Post-Grado de la Facultad de Educación de la Universidad Peruana Cayetano Heredia y de la Universidad Andina Simón Bolivia de Ecuador. Correo electrónico: ccrespoburgos@yahoo.com.mx

Fecha de recepción: 24 de febrero 2015 / Fecha de aprobación: 3 de abril de 2015.

\section{Resumen}

El mundo vive una crisis global. En este contexto, la investigación social busca los nuevos sentidos de la educación para la igualdad y la inclusión. Este documento menciona los cambios profundos planteados por diversos actores sociales y educativos en América Latina sobre el tema. Durante la última década, en esta región, las nuevas experiencias se han desarrollado para revitalizar la educación pública como un derecho humano. Las nuevas generaciones exigen una educación como derecho. Los pueblos indígenas proponen, también, otro enfoque de educación. Una educación para redescubrir un nuevo significado de la vida, para contribuir al Buen Vivir dentro de un horizonte hacia una nueva espiritualidad. Estas alternativas se exponen en este trabajo desde diferentes enfoques, debates y perspectivas, destacando el rol protagónico de los docentes en este contexto de transformaciones.

Palabras clave:

Nuevos sentidos de la educación, alternativas de desarrollo, pertinencia cultural.

\section{Summary}

The World lives a global crisis. At this context, the social research seeks the new meanings of education for the equality and inclusion. This paper mentions the deep changes raised by several social and educational actors in Latin America about this issue. In this region, new experiences have been developed in the last decade to revitalize the public education as a human right. The new generations demand an education as right. The young people not want more education as business. The indigenous peoples propose, also, other education. An education to rediscover a new meaning about of the life. A new education seeks a horizon towards a new spirituality. These alternatives are exposed in this paper from different approaches, debates and perspectives from several authors.

\section{Key words:}

New meanings of the education, Development alternatives, Cultural relevance.

\section{Resumo}

O mundo vive uma crise global. Neste contexto, a investigação social procura os novos sentidos da educação para a igualdade e a inclusão. Este documento menciona as mudanças profundas propostas por diversos atores sociais e educativas em América Latina sobre o tema. Nesta região, as novas experiências desenvolveram-se n última década para revigorar a educação pública como um direito humano. As novas gerações exigem uma educação como direito. Os jovens não querem mais educação como negócio. Os povos indígenas propõem, também, outro tipo de educação. Uma educação para redescubrir um novo significado da vida. Uma nova educação procura um horizonte para uma nova espiritualidad. Estas alternativas expõem-se neste trabalho desde diferentes enfoques, debates e perspectivas de vários autores.

Palavras chave:

Sentidos da Educação, Pertinência Cultural, Alternativas de Desenvolvimento. 


\section{Obscurecimiento de lo humano y agotamiento del modelo de desarrollo occidental}

La historia ha llegado a un punto en el que el hombre moral, el hombre íntegro, está cediendo cada vez más espacio, casi sin saberlo [...] al hombre comercial, el hombre limitado a un solo fin. Este proceso, asistido por las maravillas del avance científico, está alcanzando proporciones gigantescas, con un poder inmenso, lo que causa el desequilibrio moral del hombre y oscurece su costado más humano bajo la sombra de una organización sin alma.

Tagore, R. Citado en Nusbaum, 2010, p. 7.

A comienzos del siglo XX, Tagore alertaba sobre el obscurecimiento de lo humano al que había arribado la sociedad, con los peligros a los que conduciría este "desequilibrio moral" que se imponía en el mundo:

[...] al hacer uso de las posesiones materiales, el hombre debe tener cuidado de protegerse frente a la tiranía [de ellas]. Si su debilidad lo empequeñece hasta poder ajustarse al tamaño de su disfraz exterior, comienza un proceso de suicidio gradual por encogimiento del alma (Tagore, R. Citado en Nusbaum, 2010).

Un siglo más tarde de Tagore, el Nobel de Literatura chino 2012, Gao Xingjian, enfatizaba que nos encontramos: “[...] en una crisis no solo económica y financiera, sino también social y de pensamiento, porque hemos quedado estancados en las ideologías del siglo XX” (De Casas, 2014).

En el pensamiento crítico latinoamericano, un punto de consenso es que la serie de crisis actuales -económico-financiera, ambiental, climática, alimentaria, energética, incluso la misma crisis epistemológica- no es simplemente coyontural, sino que se trata de una crisis civilizatoria. Es decir, el modelo de desarrollo impulsado por Occidente desde hace unos cinco siglos está llegando a su punto de agotamiento, poniendo incluso en peligro la sobrevivencia de gran parte de la humanidad y de las especies vivientes. Son falsas muchas de las premisas esenciales del marco de la civilización occidental para explicar la naturaleza y dinámica de la realidad, y para justificar y legitimar sus rela- ciones asimétricas de poder con otras civilizaciones y pueblos "no-occidentales" (Souza, 2010).

Las críticas al desarrollo inequitativo del mundo y el deterioro ambiental del planeta, así como la búsqueda de nuevas vías y respuestas se multiplicaron: “[...] después de siglos de la idea de progreso y décadas de la idea de desarrollo, la humanidad nunca estuvo tan desigual y el planeta tan vulnerable" (Souza Silva, 2012).

Al celebrar el comienzo de una nueva era, los Pueblos Mayas presentaron también su crítica sobre los efectos nocivos que ha dejado la idea de desarrollo impuesta por Occidente:

La idea de progreso tan fundamental en el pensamiento hegemónico, y la pretensión de dominar mejor las fuerzas de la naturaleza para disponer de mayor bienestar, igualmente se encuentra en conflicto. Es más, la idea de desarrollo siempre creciente de las fuerzas productivas, ya no se ve, tampoco, como algo sencillo, ni mucho menos factible. Cada día que transcurre, se hace evidente que este desarrollo no mejora la calidad de vida, ni produce mayor "cuota" de felicidad. Por el contrario, deteriora la convivencia y crea tensiones insoportables como el cronometraje y la estandarización de la vida (Matul, en Souza Silva, 2012, p. 2).

Los Pueblos Mapuche denunciaron, por su parte, que han sido convertidos en refugiados del desarrollo:

[...] nuestros territorios están siendo inundados, talados nuestros bosques, contaminados nuestros suelos. Nuestros antiguos nos enseñaron que estar bien es alcanzar la armonía, mientras que la cultura dominante habla del "bien estar" como la acumulación de bienes. La cultura dominante habla de explotar los recursos. Sabemos que cuando algo explota no queda nada, nosotros hablamos de relación circular y armónica con la naturaleza y desde nuestro saber ancestral nos comprometemos a defender la diversidad biológica y cultural, el territorio y la identidad. En suma, nos comprometemos a defender la Vida no solo para nosotros sino para las generaciones futuras (AMARC, ALER, 2005). 


\section{Pérdida del sentido público de la Educación}

[...] una crisis que pasa prácticamente inadvertida, como un cáncer y que con el tiempo puede llegar a ser mucho más perjudicial para el futuro de la democracia: la crisis mundial en materia de educación.

Nusbaum, 2010.

Concomitante a Tagore, el pedagogo norteamericano John Dewey (2004) explicó en 1915 que el logro: "viene a equivaler a la clase de cosas que una máquina bien planeada puede hacer mejor que un ser humano, y el efecto principal de la educación -la construcción de una vida plena de significación- queda al margen". Luego de un siglo de vigencia, el paradigma positivista ha mostrado su agotamiento al haber convertido a la educación en un "hecho económico", con su consecuente pérdida de sentido, y tenerla prisionera de la ideología del pragmatismo, que solo busca resultados medibles y respuestas rápidas Goutier (2011), Aguilar y Bize (2010).

En las últimas décadas del siglo pasado, la óptica mercantilista se impuso en las políticas educativas en América Latina, apuntando a la autonomía en la formación individual y a una reducción de la educación al conocimiento técnico y funcional del saber. La banca multilateral difundió con valor de verdad que la inversión en educación lleva a la acumulación de capital humano, considerado un factor clave para el crecimiento económico sostenido y el aumento de los ingresos (Venegas y Mora, 2003).

Dentro de América Latina, el utilitarismo en la educación se impuso y se institucionalizó en Chile con valor de modelo, bajo el entendimiento de que es posible someter a los seres humanos a medidas estándares, a pesar de la diversidad y complejidad de las vidas individuales, en donde las diferencias tienen un punto de traducción en equivalencias (Goutier, 2011). Como consecuencia, la calidad educativa se ha centrado en torno a los resultados de aprendizaje, perdiendo su pertinencia e imposibilitando la construcción de proyectos curriculares desde la comunidad. La educación, de ser un derecho, se ha transformado en una mercancía, quedando a merced de enormes intereses empresariales. Lo paradójico es que este modelo se ha exportado al resto de nuestros países hermanos sin cuestionamiento alguno (Assaél, et al., 2011).

EducAción y CIUdAd No 29 Julio - DiciemBre de 2015 ISSN 0123-0425 - PP 40 - 48

\section{Necesidad de una transformación del modelo de pensamiento}

\author{
[...] la colonialidad del poder hace de América Latina un es- \\ cenario de des/encuentros entre nuestra experiencia, nuestro \\ conocimiento y nuestra memoria histórica.
}

Aníbal Quijano.

Vivimos la emergencia de un momento histórico sin precedentes en el que, tal vez por primera vez, en más de 500 años de subordinación, silencio y colonialismo, emerge la posibilidad de otro horizonte de sentido histórico.

Quijano, 2010.

Se ha reconocido que la superación del enfoque del desarrollo y de la educación requiere de una transformación profunda del modelo de pensamiento que los sustenta (Morin, 2005). A propósito, el Nobel de Literatura chino de 2012, Gao Xingjian, demandaba a los intelectuales de todo el mundo abordar la realidad y poner en marcha un nuevo pensamiento, un nuevo renacimiento, que tiene que ser el punto de partida y no debe nacer en un país, puesto que al tratar problemas universales tiene que ser global (De Casas, 2014).

Morin (2012) ha explicado la necesidad de una reforma del conocimiento, que a su vez exige una reforma del pensamiento: "Nuestro modo de conocimiento no ha desarrollado suficientemente la aptitud para contextualizar la información e integrarla en un conjunto que le dé sentido". Refiriéndose al desarrollo de la aptitud para organizar el pensamiento por parte de todo ciudadano del nuevo milenio, consideró indispensable una reforma del pensamiento que sea paradigmática y no programática (2005), reforma que tenga un carácter epistemológico y reflexivo (2012). ¿Cómo percibir y concebir el contexto, lo global (la relación todo/partes), lo multidimensional, lo complejo?

La educación del futuro está enfrentada a un problema universal de pertinencia en el conocimiento, porque hay una "inadecuación” cada vez más amplia, profunda y grave; por un lado, entre nuestros saberes desunidos, divididos y compartimentados y, por el otro, realidades o problemas cada vez más polidisciplinarios, transversales, multidimensionales, transnacionales, globales, planetarios. 
Nosotros, los habitantes del mundo occidental u occidentalizado, sufrimos, sin ser conscientes de ello, dos tipos de carencias cognitivas: la ceguera propia de un modo de conocimiento que, al compartimentar los saberes, desintegra los problemas fundamentales y globales que exigen un conocimiento interdisciplinar; el occidentalcentrismo, que nos coloca en el trono de la relacionalidad y nos da la ilusión de poseer lo universal (Morin, 2012, p. 19).

La transformación profunda de los modelos de pensamiento pasa, entre otros aspectos, por rescatar y renovar filosofías y conocimientos distintos del hegemónico occidental, y por establecer un diálogo intercultural como base de un mundo conformado por varias racionalidades, saberes, modos de producir sentido y explicar el mundo, cuya base común puede constituir los enfoques relacionales y el aprendizaje mutuo, en un marco de reconocimiento de las diversidades (Burch, 2014).

Adentrados en el siglo XXI presenciamos las semillas culturales de cambios profundos en gestación, en el marco de la gran crisis cultural de la modernidad occidental. Nuevos pensamientos y sensibilidades buscan expresarse en la sociedad y la educación, alentados por la diversidad de los pueblos y la presencia de nuevos actores sociales.

\section{Nuevos sentidos para la educación}

En la región latinoamericana se asiste a la emergencia de nuevos paradigmas sobre la sociedad y el desarrollo, así como al surgimiento consecuente de nuevos sentidos para la educación, en medio de la persistencia de rasgos de los modelos educativos implantados en las últimas décadas del siglo XX, bajo enfoques neoliberales.

Se viven en la región experiencias inéditas de revitalización de lo público y también de presencia pública de nuevas generaciones que no quieren más la educación como lucro, por sus efectos de inequidad y exclusión (Le Monde, 2003). Surge a la vez el postulado de impulsar políticas y estrategias de ciencia y tecnología con criterio de "autodeterminación nacional y regional" (Dussel, 2014), dado su carácter de mediación esencial para el desarrollo y la riqueza de un país, no solo cuantitativa, sino cualitativa, del desarrollo de la vida concreta de una población cultural, diversa e históricamente sometida a la exclusión e inequidad.
En estos nuevos escenarios se reconecta a la educación con demandas históricas de sociedades interculturales y diversas, y se busca dar respuesta a la necesidad de igualdad de oportunidades para todas y todos. Se presentan, en consecuencia, para la educación numerosos retos, puesto que las transformaciones requeridas están ocurriendo en escenarios en disputa, por la herencia de más de dos décadas de aplicación de agendas externas de política educativa. Se abren a la vez insospechadas posibilidades de construcción de nuevos sentidos y respuestas educativas enriquecidas por diversidades que surgen desde los países y pueblos (Crespo, 2013).

Rigoberta Menchú (2000), líder indígena guatemalteca, premio Nobel de la Paz, destacó la contribución que ofrecen los pueblos indígenas de América para el desarrollo de la humanidad:

\begin{abstract}
Dentro de sus valores profundos, las culturas originarias poseen y cuidan concepciones de gran importancia para la conformación de una idea nueva y valedera del desarrollo sostenible: el ser humano como eje y sujeto fundamental del desarrollo; la comunidad como generadora de iniciativas y dinámicas para la solución de los problemas comunes; la preeminencia de los intereses de la comunidad sobre los individuales; la convivencia armónica entre el ser humano y la naturaleza; la ética como regla fundamental para la relación entre las personas y entre sus comunidades y otras.
\end{abstract}

Los pensamientos de los pueblos originarios ofrecen orientaciones fundamentales para imaginar nuevos modos de convivencia social y nuevos sentidos para la educación. Los pueblos originarios nos brindan premisas para la "con-vivencia" armónica entre todas las formas y modos de vida humana y no humana.

En los inicios de la nueva era de la Civilización Maya, sus sabios han invitado a la humanidad a "ensoñar la trama de la vida" con una pregunta fundamental: ¿Qué es más importante que la vida? Su respuesta es categórica: "nada [puesto que] la vida es el origen, centro y fin de todo el pensar y actuar humano". Su sabiduría sobre la vida y la educación puede apreciarse en los siguientes segmentos:

Se trata de comprender la vida como búsqueda de sentido en $[\ldots]$ nuestras relaciones, en lo que hacemos y en lo que dejamos de hacer, en lo que somos y en lo que quisiéramos ser. Ese conocimiento integra el sentir, el saber, el vivir [...] nada debe 
hacer quien aprende si a eso que tienen que hacer no le encuentra sentido, dicen los mayores (Matul, en Souza, 2012).

Educar y educarse es, en consecuencia, encontrar sentido a la propia vida, descolonizándonos de la razón y buscando nuestra conciencia cósmica; como experiencia compleja, significa volver a la tierra fecunda con mitos, leyendas, tradiciones, ritos, cuentos y poesía. Igualmente, encarna la comprensión del propósito espiritual del universo, en el ánimo de recuperar el simbolismo del hombre como hijo de la inmortalidad y nexo de todo cuanto existe.

Por otra parte, los pueblos originarios de Bolivia han incorporado en la nueva Constitución (República de Bolivia, 2008) el compromiso del Estado de asumir y promover los principios ético-morales de la sociedad plural: "ama qhilla, ama llulla, ama suwa" (no seas flojo, no seas mentiroso, ni seas ladrón); "suma qamaña" (vivir bien); "ñandereko" (vida armoniosa); "teko kavi" (vida buena); "ivi maraei" (tierra sin mal) y "qhapaj ñan” (camino o vida noble)".

En el contexto de crisis de la hegemonía del neoliberalismo, en varios países de América Latina se han generado rupturas con los parámetros homogéneos impuestos por la "globalización" del capital. Estas transformaciones responden a un momento histórico de cambio social y político que transita la región latinoamericana, donde tanto las actuales Constituciones, como las recientemente aprobadas leyes educativas, recolocan la educación desde nuevos paradigmas sociales, no en clave de "productividad", como caracterizó a las políticas de los 90, sino del "Buen Vivir" o el "Vivir Bien", inspiradas en la construcción de nuevas sociedades que cuestionan el concepto lineal de desarrollo y progreso como acumulación y consumo.

Se trata de un cambio de paradigmas y no de simples reformas o cambios de contenidos (Huanacuni, 2010), que cuestiona el modo de acumulación capitalista, el sistema socio-económico y político vigente y el tipo de relaciones sociales (Larrea, 2010). Estas nuevas perspectivas epistemológicas se nutren de las concepciones de los pueblos ancestrales, de las luchas emancipadoras y de las teorías críticas.

En este contexto, Bolivia ha propuesto la construcción del Estado plurinacional y, para ello, ha puesto en marcha un modelo educativo al que ha denominado "socio comunitario productivo", que se plasma en propuestas como la descolonización del pensamiento, la convivencia en la diversidad, el pluralismo epistemológico y el aprendizaje comunitario.
Para el contexto actual de América Latina, el pensador brasileño José Souza Silva (2012) ha enfatizado la necesidad de una Pedagogía intercultural crítica. Se pregunta: ¿cómo revolucionar la pedagogía para cambiar el estado actual de cosas desde el proceso educativo? La respuesta es la utopía de instituir la "pedagogía de la vida", para lograr la felicidad para todos, en el contexto de una sociedad que inagurará "el día después del desarrollo":

\begin{abstract}
No será fácil superar este reto que exige imaginación pedagógica crítica para desentrañar el vínculo geopolítico e histórico entre la idea de raza y la relación poder-saber-ser-naturaleza que condiciona las relaciones internacionales, la dinámica del Estado y la naturaleza de los sistemas de educación, comunicación, cooperación e innovación, así como para asumir las premisas que emergen en América Latina para construir el paradigma del "buen vivir" / "vivir bien". Una opción es instituir "la vida" como fuente y fin de la educación, comunicación, cooperación e innovación y reemplazar el "ser desarrollado", como meta, por el "ser feliz" como fin.
\end{abstract}

La Pedagogía intercultural crítica significa avanzar hacia un desarrollo humano, social y espiritual colectivo, tratando a los humanos como seres sociales, culturales y espirituales que aprenden en interacción interétnica a través del diálogo intercultural y democrático, entre ellos y desde su contexto material, histórico, social, cultural y espiritual. En la interculturalidad están en juego no solo paradigmas, cosmovisiones, miradas diversas de las culturas, sino eminentemente un proceso político, social, económico y cultural de cambio profundo, que replantea modelos de vida, desarrollo y medio ambiente; son relaciones hacia una nueva cultura que retoma saberes de las culturas originarias en su relación armónica con la naturaleza y la sociedad, en elementos profundos de solidaridad, en la centralidad de la "Pacha Mama" como sujeto de derechos y de construcción de un proyecto sostenible de desarrollo (Souza Silva, 2012).

Por su parte, el biólogo y filósofo chileno Humberto Maturana (2009) ha argumentado que si queremos un nuevo paradigma, lo central en el tema de la educación no está en los contenidos sino en la emoción desde donde se hace la educación. Si pretendemos que los niños crezcan como ciudadanos, como seres éticos, responsables, que posean un vivir digno, estético, grato y creativo, en el sentido de que tengan imaginación, que tengan presencia para el bienestar, hay que preocuparse de las emo- 
ciones. Maturana considera que las emociones han sido tratadas como distorsiones de la razón, que se dejó de entender su presencia y su importancia, ciegos a los fundamentos emocionales de lo que hacemos, referencia de cómo actuamos.

Desde otro lenguaje y cosmovisión, los pueblos Mayas han sintetizado con mucha claridad su visión pedagógica desde una perspectiva integradora de la vida, que se alcanza ensanchando la razón desde una conexión con su sentido espiritual:

[Nuestra visión pedagógica] no puede continuar con el pensar calculador colmado en números y datos, tampoco podemos perpetuar nuestra evasión a la definición ontológica de nuestras naciones. [Debemos superar] los principios mecanicistas del siglo XVIII y volver al encaje con la familia, la sociedad, la historia, el aprendizaje, la creatividad, los sentimientos, las cosmogonías y la naturaleza [...] volver a la inspiración individual y colectiva que solo puede aportar el sentido espiritual más ennoblecido, al que podemos llegar ensanchando la razón por la vía de la pregunta, del misterio, de lo sagrado y de la pasión (Matul, en Souza, 2012, p. 4)

Hoy estamos experimentando una nueva manera de entender e interpretar la vida. Este innovador conocimiento lleva consigo un cambio $[\ldots]$ completo de aquella vieja visión de la vida que separa a los seres humanos del universo, al tiempo que fractura el espíritu de la materia [...] Somos parte de la Tierra, conformamos una sola unidad que nos obliga a ser, vivir, compartir, comunicar y comulgar con ella, como seres vivos creadores y recreadores de la vida (Matul, en Souza, 2012, p. 8) ${ }^{1}$.

Nos enriquecemos con la sabiduría y las raíces ancestrales de nuestros pueblos originarios, y afirmamos nuestro interés mayor por atender a un futuro humano, que apenas se perfila en el horizonte de la vieja civilización que hemos heredado. Las nuevas sensibilidades y la conciencia colectiva de los pueblos buscan construir un nuevo mundo en unidad, buscan una puerta hacia el futuro sin contradicción, sin violencia, sin más sufrimientos sociales, sin más fracturas al espíritu. Las alternativas que se

1 Poema Galáctico: Cuenta del tiempo cultural Maya. Citado en José de Souza Silva. La pedagogía de la felicidad en una educación para la vida. El paradigma del "buen vivir"/ "vivir bien" y la construcción pedagógica del "día después del desarrollo". Campina Grande-PB, Brasil. Septiembre de 2012. vislumbran frente a la realidad social actual requieren ser visibilizadas, profundizadas, difundidas y teorizadas, como nuevas semillas.

\section{El protagonismo docente}

En el contexto de estos nuevos pensamientos y escenarios que se viven en la región, la formación y el trabajo docente ocupan un lugar central en los debates sobre la calidad educativa, las reformas y los proyectos educativos nacionales. Las transformaciones educativas son posibles con docentes formados con una capacidad crítica y condiciones para dirigir también su desarrollo, en la convivencia armónica con los demás y la naturaleza, en la comprensión de un nuevo rol histórico para implementar dimensiones pedagógicas desde la construcción viva de la interculturalidad (Robalino y Crespo, 2014)

La producción de conocimientos, por parte de los docentes como actores de las experiencias educativas, supone que la realidad no se asume como una objetividad dada y externa al sujeto, sino como una construcción social a partir de la perspectiva y significados de quienes la viven. Este es el caso de la "Sistematización de experiencias" (significativas) o de Buenas Prácticas, modalidad de investigación que contempla un enfoque abierto a la generación de conocimiento intercultural y que reconoce el valor del saber común producido desde la vida cotidiana y desde distintos tipos de saberes locales producidos desde la cultura (Geertz, 1994).

La práctica transformadora de los docentes se nutre con las contribuciones del paradigma alternativo en el campo de la metodología y de la concepción de la realidad que se gestó en América Latina a partir de la década del 60. La idea central alrededor de la cual se cristalizó lo que pudiera considerarse como base de este paradigma alterno, fue la posibilidad de crear y poseer conocimiento científico desde la propia acción social de las poblaciones subalternas u oprimidas, según lo comprendió Paulo Freire en su Pedagogía del Oprimido (1970).

Se vinculó investigación social con la acción social y política, posibilitando una síntesis e influencia mutua para aumentar, tanto el nivel de eficacia de la acción, como el entendimiento de la realidad (Fals, 1978). Este precursor de la investigación-acción en América Latina, destacó que, dado que el criterio de la corrección del pensamiento es la realidad", el último criterio de validez del conocimiento científico venía a ser, entonces, la 
"praxis", entendida como una unidad dialéctica formada por la teoría y la práctica, en la cual la práctica es cíclicamente determinante.

En la actualidad se requiere de abrir un amplio debate para construir una comprensión sobre calidad de la educación, coherente con los nuevos paradigmas de desarrollo que se encuentran en construcción en nuestras sociedades latinoamericanas. Estos son temas de base para la reflexión sobre un nuevo sistema de formación de profesores, que aspira a promoverlos como sujetos generadores de conocimiento (Robalino y Crespo, 2014).

La revalorización epistemológica contemporánea del pensamiento común, implica la recuperación, para la educación, del valor y el significado de los saberes construidos desde las culturas de los pueblos, particularmente de los pueblos originarios. Para la gestación de otra educación adquiere importancia central la integralidad de estos saberes y conocimientos, así como el valor de las dimensiones de intra e interculturalidad que incluyen aspectos como los conocimientos y saberes originarios; el pensamiento desde la intraculturalidad; la educación desde la interculturalidad y el reencuentro de los saberes (Ministerio de Educación del Estado Plurinacional de Bolivia, 2009). 


\section{Referencias}

Aguilar, Mario y Bize, Rebeca. (2010). Pedagogía de la Intencionalidad. Educando para una conciencia activa. Santiago: Virtual ediciones.

AMARC-ALER. (2005). El manualito ambiental. Buenos Aires: ALER.

Assaél, J., Cornejo, R., González, J., Redondo, J., Sánchez, R., y Sabarzo, M. (2011, Abril-Junio). La empresa educativa chilena. Educacao e Sociedade. Revista de Cencias da Educacao. V. 32, No. 115, pp. 287 - 304. Campinas.

Burch, S. (2014). Diálogo de saberes. América Latina en movimiento 493. Ciencia, Tecnología e Innovación. ALAI.

Crespo, Carlos. (2013). Escenarios en disputa, nuevos sentidos para la educación y el trabajo docente: transformaciones educativas en Ecuador (Sumak Kawsay) y Bolivia (Sumaq Qamaña). Ponencia presentada en el II Encontro Luso-Brasileiro sobre o trabalho docente e formacao. "Políticas, práticas e investigacao: pontes para uma mudanca". 1 al 3 de noviembre 2013. Universidad de Porto.

De Casas, Ernesto. (2014-Abril). Un nuevo pensamiento, un nuevo renacimiento. Pressenza. Agencia internacional Noticias. Obtenido desde www.pressenza.com/es

Dewey, John. (2004). Democracia y Educación. Madrid: Morata.

Dussel, E. (2014). Hacia la liberación científica y tecnológica. América Latina en Movimiento 493. Ciencia, Tecnología e Innovación. ALAI.

Estado Plurinacional de Bolivia. (2007). Constitución Política del Estado. La Paz: Publicaciones Ministerio de Educación.

Fals Borda, Orlando. (1978). Por la praxis: el problema de cómo investigar la realidad para transformarla. Bogotá: Federación para el análisis de la realidad colombiana (FUNDABCO).
Freire, Paulo. (1970). Pedagogía del Oprimido. Montevideo: Paz e Terra.

Geertz, C. (1994). El sentido común como un sistema cultural. Conocimiento local. Barcelona: Paidós.

Goutier, Emilio. (2012). Ideas acerca de la educación de los estudiantes chilenos movilizados. Conferencia presentada en el I Seminario Internacional sobre Trabajo Docente en América Latina, organizado por SINTEPE. Octubre, 2012. Recife, Brasil.

Huanacuni Mamani, Fernando. (2010). Vivir Bien/Buen Vivir. Filosofía, políticas, estrategias y experiencias regionales. La Paz: Ediciones Convenio Andrés Bello.

Larrea, Ana. (2010). La disputa de sentidos por el Buen Vivir como proceso. Socialismo y Sumak Kawsay. Los nuevos retos de América Latina. Quito: SENPLADES.

Le Monde Diplomatique. (2003). La Educación no es una mercancía. Santiago: Editorial Aún creemos en los sueños.

Maturana, Humberto. (1999). Transformación en la Convivencia. Santiago: Dolmen.

Menchú, Rigoberta. (2000). El desarrolllo sostenible. Requisito para la pervivencia de la humanidad. El vuelo de la serpiente. Desarrollo sostenible en la América Prehispánica. Bogotá: Siglo del Hombre Editores-Unesco.

Ministerio de Educación del Estado Plurinacional de Bolivia. (2009). Primer Simposio Internacional de Educación Intercultural del Abya Yala. Memoria 2009. La Paz: UNESCO, UNICEF, EIBAMAZ.

Morin, Edgar. (2005). Siete saberes necesarios para la educación del futuro, Quito: UNESCO-Fundación Santillana. 
Morin, Edgar. (2012). La vía para el futuro de la humanidad. Barcelona: Espasa Libros.

Nussbaum, Martha. (2010). Sin fines de lucro. Por qué la democracia necesita de las humanidades. Buenos Aires: Katz Editores.

Robalino, M., y Crespo, C. (s.f.). Repensar las sociedades, repensar la formación de los docentes. Revista Praxis Educacional da Universidade Nacional do Estado de Bahía. Dossiê "Trabalho docente e desenvolvimento profissional do professor”. Bahía, Brasil.

Quijano, Aníbal. (2010). América Latina: hacia un nuevo sentido histórico. En León, Irene. (Coord.). Sumak Kawsay/Buen Vivir y cambios civilizatorios (pp. 55-71). Quito: FEDAEPS.

Souza Silva, José. (2012-Septiembre). La pedagogía de la felicidad en una educación para la vida. El paradigma del "buen vivir"/"vivir bien" y la construcción pedagógica del "día después del desarrollo". Campina Grande-PB, Brasil.

Souza, J. (2010). La investigación científica para el desarrollo y el desarrollo de la investigación científica. Geopolítica del conocimiento y los aportes de la ciencia a la gestión del "desarrollo" en América Latina. Campina Grande, Brasil. 